\title{
Is Acromial Fracture after Reverse Total Shoulder Arthroplasty a Negligible Complication?: A Systematic Review
}

\author{
Chul-Hyun Cho, MD, Jae-Won Jung, MD, Sang-Soo Na, MD, Ki-Cheor Bae, MD, Kyung-Jae Lee, MD, \\ Du-Han Kim, MD
}

Department of Orthopedic Surgery, Dongsan Medical Center, Keimyung University School of Medicine, Daegu, Korea

Background: The purpose of this study was to investigate the incidence of acromial fracture after reverse total shoulder arthroplasty (RTSA) and clinical and radiological outcomes of treatment of the fracture.

Methods: A systematic review was performed to identify studies that reported the results of treatment of acromial fractures after RTSA. A literature search was conducted by two investigators using four databases (PubMed, Embase, Cochrane, and Ovid Medline).

Results: Fifteen studies (2,857 shoulders) satisfied our inclusion criteria. The incidence of acromial fracture after RTSA was $4.0 \%$ $(114 / 2,857)$. The mean age of the patients at the time of fracture was 72.9 years (range, 51 to 91 years). The mean time from RTSA to diagnosis of acromial fracture was 9.4 months (range, 1 to 94 months). One hundred shoulders (87.7\%) were treated conservatively and 14 shoulders (12.3\%) were treated surgically. The mean follow-up period after acromial fracture was 33.8 months. The overall union rate was $50.0 \%$ (43.8\% for conservative treatment and $87.5 \%$ for operative treatment). The fracture incidence was significantly different among the medial glenoid and medial humerus prosthesis design (8.4\%), the lateral glenoid and medial humerus design (4.0\%), and the medial glenoid and lateral humerus design (2.8\%). The mean values at final follow-up were as follows: visual analog scale score, 2.2; American Shoulder and Elbow Surgeons score, 59.1; Constant score, 59.7; and Simple Shoulder Test, 5.8. The mean forward flexion, abduction, and external rotation were $102.3^{\circ}, 92.3^{\circ}$, and $25.8^{\circ}$, respectively.

Conclusions: Acromial fractures after RTSA are a complication neither uncommon nor negligible. In the absence of studies with high-level evidence, there is a controversy on the outcomes after treatment. Further well-designed prospective randomized controlled studies with a long-term follow-up should be performed to ascertain the diagnosis, treatment, and prognosis of acromial fractures after RTSA.

Keywords: Reverse shoulder arthroplasty, Acromion, Scapular, Fracture, Systematic review

Reverse total shoulder arthroplasty (RTSA) is an innovative surgical technique that can compensate the force of

Received February 7, 2019; Accepted March 8, 2019

Correspondence to: Du-Han Kim, MD

Department of Orthopedic Surgery, Dongsan Medical Center, Keimyung University School of Medicine, 56 Dalseong-ro, Jung-gu, Daegu 41931, Korea

Tel: +82-53-250-8160, Fax: +82-53-250-7205

E-mail: osmdkdh@gmail.com the deltoid muscle when the rotator cuff does not function. The mechanical principle of RTSA, which was invented by Grammont and Baulot, ${ }^{1)}$ is medialization of the center of rotation relative to the native level of the glenoid to allow for a greater deltoid lever arm at the start of shoulder movement. ${ }^{2,3)}$ Although RTSA has been associated with significant improvement in pain and function, many complications have been reported, including scapular notching, fixation failure, infection, instability, glenoid component loosening, nerve injury, and acromial fracture. ${ }^{4,5)}$ 
Cho et al. Acromial Fracture after Reverse Shoulder Arthroplasty: A Systematic Review

Clinics in Orthopedic Surgery • Vol. 11, No. 4, $2019 \bullet$ www.ecios.org

The reported incidence of acromial fractures after RTSA ranges from $0.6 \%$ to $15.8 \%{ }^{6-21)}$ Because of the longer arm length and greater deltoid tension after RTSA, the force on deltoid muscle origin increases. Thus, fractures after RTSA can occur at various locations from the acromion to the scapular spine. ${ }^{14,21)}$ Since RTSA has been introduced recently, the management of postoperative acromial fractures is not well established. ${ }^{19)}$

Crosby et al. ${ }^{21)}$ classified acromial fractures into three distinct patterns: (1) type I, avulsion fractures of the anterior acromion; (2) type II, fractures of the acromion posterior to the acromioclavicular joint; and (3) type III, scapular spine fractures. They proposed that type I fractures can be treated conservatively, whereas unstable type II and type III fractures require open reduction and internal fixation (ORIF). Levy et al. ${ }^{14)}$ reclassified acromial fractures into three types on the basis of the degree of deltoid origin involvement and recommended nonoperative treatment regardless of fracture type. Until now, there have been no clear treatment guidelines for acromial fractures after RTSA. Furthermore, there has been controversy regarding the outcomes of conservative or operative treatment of acromial fractures. $2,7,10,13,14,16,17,20)$

Acromial fractures after RTSA are challenging for the orthopedic surgeon to diagnose and manage, and this may lead to functional deterioration in the long term. ${ }^{22)}$ Acromial fractures after RTSA can be either nondisplaced or displaced fractures. Nondisplaced fractures, if detected early, can be treated conservatively, whereas management of displaced fractures with delayed or neglected diagnosis is challenging because of high rates of malunion or nonunion, functional deterioration, and inconsistent results after operative treatment. ${ }^{23)}$

Although acromial fractures are a recognized complication after RTSA, the incidence, treatment methods, and outcomes are poorly understood. To the best of our knowledge, there has been no systematic review on acromial fractures after RTSA. The purpose of this study was to investigate the incidence of acromial fractures after RTSA and clinical and radiological outcomes of treatment through a systematic review.

\section{METHODS}

A systematic review was performed in accordance with Preferred Reporting Items for Systematic Reviews and Meta-Analyses (PRISMA) guidelines.

\section{Literature Search}

Articles were retrieved from four computerized literature databases (PubMed, Embase, Cochrane library, and Ovid Medline), using the following search terms: "reverse total shoulder arthroplasty" OR "prosthesis" OR "replacement" or "acromion fracture" OR "scapular fracture." All articles not pertaining to the shoulder were excluded. Considering the year of RTSA introduction, we limited the period of and Ovid Medline)

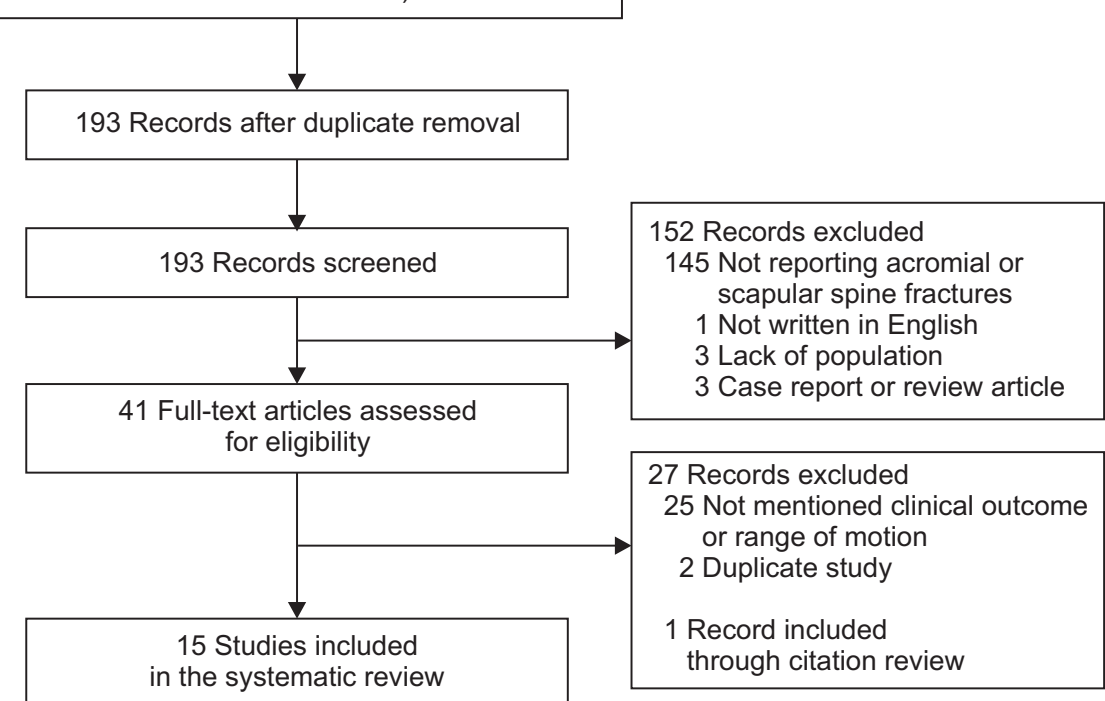

Fig. 1. Flow diagram for the systematic review process. 
Cho et al. Acromial Fracture after Reverse Shoulder Arthroplasty: A Systematic Review

Clinics in Orthopedic Surgery • Vol. 11, No. 4, 2019• www.ecios.org

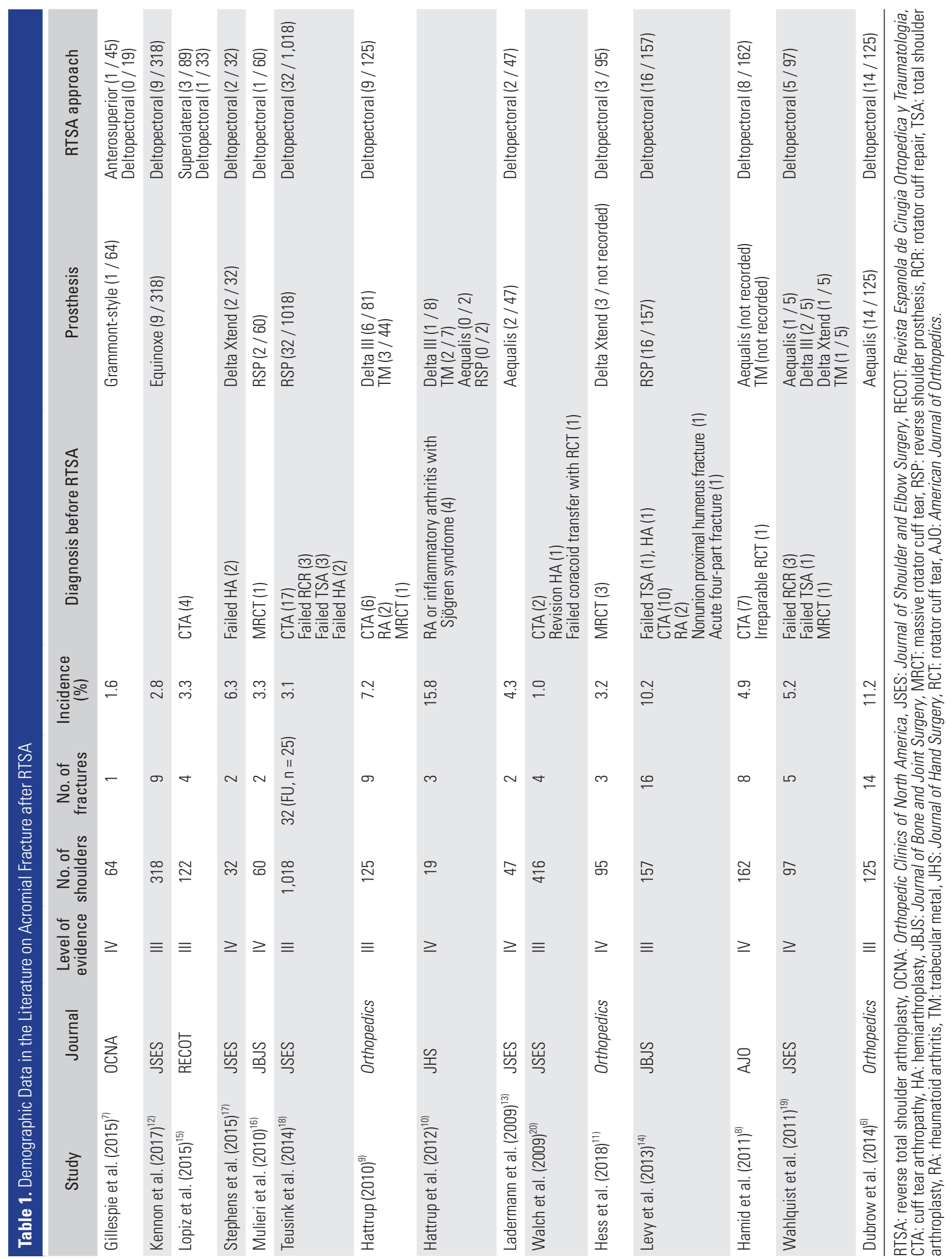


Cho et al. Acromial Fracture after Reverse Shoulder Arthroplasty: A Systematic Review

Clinics in Orthopedic Surgery • Vol. 11, No. 4, 2019• www.ecios.org

publication to 2000-2018. The search was performed by two independent investigators ( $\mathrm{CHC}$, JWJ). If there was a disagreement about the inclusion of an article, the final decision was made by a senior author (CHC).

\section{Inclusion and Exclusion Criteria}

Inclusion criteria were articles (1) written in English, (2) with a minimum 12-month follow-up after RTSA, and (3) reporting outcome data including clinical score and range of motion (ROM). Exclusion criteria were (1) scapular body fracture, (2) case report, and (3) review article.

\section{Study Selection}

The two investigators reviewed the articles separately and selected articles according to the inclusion and exclusion criteria. One investigator (JWJ) checked the full text of all selected articles and classified them in order of importance. The other investigator $(\mathrm{CHC})$ double-checked the sorted articles and approved the sorting. According to the above criteria, 234 articles were first selected, 41 of them were excluded due to duplication. Among 193 articles, 145 were excluded because acromial or scapular spine fractures were not recorded, and seven were excluded because of language other than English $(\mathrm{n}=1)$, lack of sufficient study population $(n=3)$, begin a case report or a review article $(n=3)$. A full-text review was performed for the remaining 41 articles, and 25 articles were excluded because they did not mention clinical scores or ROM of the affected shoulder. The two investigators and the senior author checked all references in each article. Two articles were excluded because they were multicenter studies and their study populations overlapped, and one article was included via citation tracking. Finally, 15 articles were included in this systematic review (Fig. 1).

\section{Statistical Analysis}

All statistical analyses were performed using IBM SPSS ver. 20.0 (SPSS Inc., Chicago, IL). The linear by linear association was used to determine the correlation between the incidence and various factors. A $p<0.05$ was considered statistically significant.

\section{RESULTS}

Among the 15 articles, seven were level III studies and eight were level IV studies according to Level of Evidence Guidelines (Tables 1 and 2). ${ }^{6-20)}$

\section{Incidence}

Among 2,857 shoulders, 114 acromial or scapular spine fractures after RTSA were found. The overall incidence was $4.0 \%$ (range, $1.0 \%$ to $15.8 \%$ ). Among 93 fractures described in 14 studies, ${ }^{7-20)}$ there were $46(49.5 \%)$ acromial fractures and 47 (50.5\%) scapular spine fractures.

\section{Demographics}

The mean age of the patients at the time of fracture was 72.9 years (range, 51 to 91 years) and the male to female ratio was 1:5.4 in 10 studies. The mean time from RTSA to diagnosis of acromial fracture was 9.4 months (range, 1 to 94 months) in 14 studies. The mean follow-up period after acromial fracture was 33.8 months in eight studies.

The incidence of postoperative acromial fractures was $4.2 \%(89 / 2,131)$ in the deltopectoral approach group in 13 studies, 2.2\% (1 / 45) in the anterosuperior approach group in one study, and $1.1 \%(1 / 89)$ in the superolateral approach group in one study. There was no statistically significant difference between the three groups $(p=0.119)$. Twelve studies mentioned the prosthesis types used for RTSA. The incidence of postoperative acromial fractures was 2.8\% (9 / 318) in patients with Equinoxe (Exactech, Gainesville, FL, USA), 8.1\% (3 / 37) in patients with Delta Xtend (DePuy, Warsaw, IN, USA), 4.0\% (50 / 1,237) in patients with the reverse shoulder prosthesis (RSP; DJO Surgical, Austin, TX, USA), 9.6\% (9/94) in patients with Delta III (DePuy), 9.5\% (17 / 179) in patients with Aequalis (Tornier, Montbonnot-Saint-Martin, France), and 10.7\% (6 / 56) in patients with TM Reverse Shoulder System (Zimmer, Warsaw, IN, USA). The incidence according to prosthesis design was $8.4 \%$ (36/430) for the medial glenoid/medial humerus (MGMH) design (Delta III, Delta Xtrend, Aequalis, TM Reverse Shoulder System), 4.0\% (50 / 1237) for the lateral glenoid/medial humerus (LGMH) design (RSP), and 2.8\% (9 / 318) for the medial glenoid/ lateral humerus (MGLH) design (Equinoxe). There was statistically significant difference among the three designs $(p<0.001)$.

\section{Treatment}

One hundred shoulders (87.7\%) were treated conservatively and 14 shoulders (12.3\%) were treated surgically. Conservative treatment usually included immobilization with an abduction brace or arm sling for 6 to 8 weeks. Operative treatment included ORIF with plating (11/ 14), ORIF with tension band wiring (2 / 14), and revision RTSA with ORIF (1 / 14). On the analysis of treatment method according to fracture location, 93.5\% (43/46) of the patients with acromial fracture had conservative treatment in nine studies and 6.5\% (3/46) had operative treatment in one study; $76.6 \%$ (36/47) of the patients 
Cho et al. Acromial Fracture after Reverse Shoulder Arthroplasty: A Systematic Review

Clinics in Orthopedic Surgery • Vol. 11, No. 4, 2019• www.ecios.org

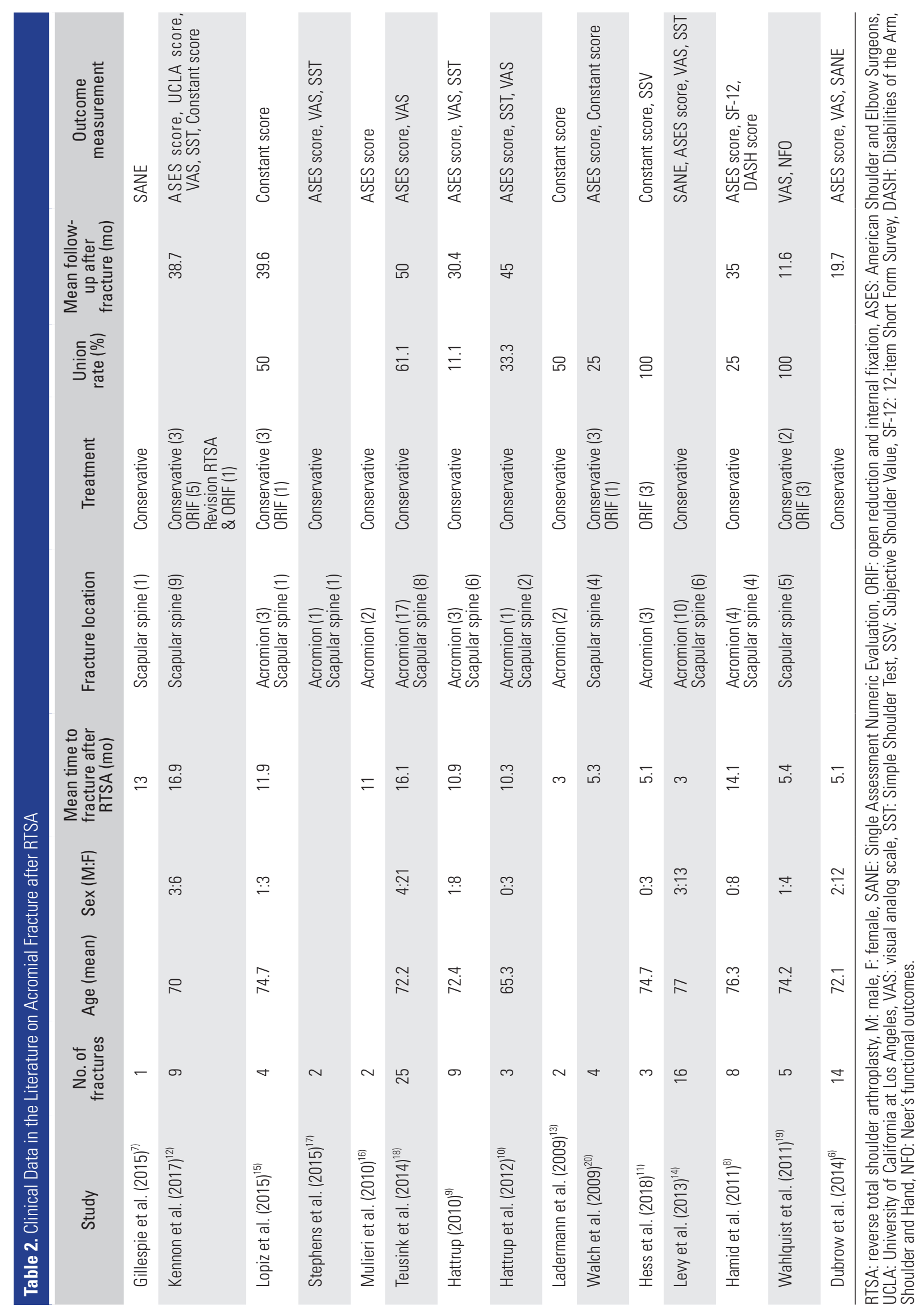


Cho et al. Acromial Fracture after Reverse Shoulder Arthroplasty: A Systematic Review

Clinics in Orthopedic Surgery • Vol. 11, No. 4, 2019• www.ecios.org

with scapular spine fracture had conservative treatment in 10 studies and $23.4 \%(11 / 47)$ had operative treatment in four studies.

\section{Outcomes}

The mean union rate after treatment of acromial and scapular spine fractures reported in nine studies was 50\% (28/ 56 ) at the final follow-up. After conservative treatment, the union rate was $48.1 \%$ (13/27) for acromial fractures and $38.1 \%$ (8 / 21) for scapular spine fractures. After operative treatment, it was $100 \%(3 / 3)$ for acromial fractures and $80 \%$ (4 / 5) for scapular spine fractures. Overall, the union rate was $43.8 \%(21 / 48)$ for conservative treatment and $87.5 \%$ ( 7 / 8) for operative treatment.

At the final follow-up after management of acromial fractures, the mean American Shoulder and Elbow Surgeons (ASES) score was 59.1 in nine studies, the visual analog scale (VAS) for pain was 2.2 in seven studies, Simple Shoulder Test was 5.8 in four studies, Constant score was 59.7 in five studies, and Single Assessment Numeric Evaluation (SANE) score was 69 in two studies (Table 3). Four studies compared scores obtained before treatment of the fracture and at the final follow-up after treatment. The mean ASES score increased from 43.8 at the first visit after fracture to 71.0 at the final follow-up in patients with conservative treatment and from 5.8 to 80.3 in patients with operative treatment. The mean VAS score decreased from 5.4 at the first visit after fracture to 1.0 at the final followup in patients with conservative treatment and from 9.3 to 0 in patients with operative treatment. The mean Constant score increased from 10.5 at the first visit after acromial fracture to 57 at the final follow-up in patients with conservative treatment and from 10.3 to 73 in patients with operative treatment.

At the final follow-up after management of the fractures, the mean active forward flexion, abduction, and external rotation were $102.3^{\circ}, 92.3^{\circ}$, and $25.8^{\circ}$, respectively. The mean forward flexion increased from $79.2^{\circ}$ at the first visit after fracture to $108.3^{\circ}$ at the final follow-up in patients with conservative treatment and from $61.5^{\circ}$ to $126.9^{\circ}$ in patients with operative treatment. The mean abduction increased from $15^{\circ}$ at the first visit after fracture to $75^{\circ}$ at the final follow-up in patients with conservative treatment and from $52.3^{\circ}$ to $107.5^{\circ}$ in patients with operative treatment. The mean external rotation increased from $-2.5^{\circ}$ at the first visit after fracture to $32.5^{\circ}$ at the final follow-up in patients with conservative treatment and from $0.8^{\circ}$ to $29.2^{\circ}$ in patients with operative treatment.

\section{DISCUSSION}

To the best of our knowledge, this is the first systematic review investigating the incidence and treatment outcomes of acromial fractures after RTSA. Our study revealed that acromial fractures after RTSA are a complication neither uncommon nor negligible. So far, there have been no clear treatment guidelines and there has been controversy regarding the outcomes of treatment because of the low level of evidence.

The incidence of acromial fractures after RTSA ranges from $0.6 \%$ to $15.8 \%{ }^{6-21)}$ In our systematic review of 15 studies, ${ }^{6-20)}$ the overall incidence was $4.0 \%$ (range, $1.0 \%$ to $15.8 \%$ ). We think that the incidence may have been underestimated; the fractures are difficult to detect in plain radiographs, and thus the diagnosis is frequently neglected. This may be one of the reasons for the large difference in the incidence among previous studies. Otto et al. ${ }^{22)}$ reported that $32.1 \%$ of patients with postoperative acromial or scapular fractures initially had unremarkable radiographs but finally were diagnosed with displaced fractures. Therefore, we believe that the reported incidence will increase, given that more attention is paid to the diagnosis and radiological assessment of acromial or scapular fractures and RTSA is more frequently performed ${ }^{23)}$ and followed by long-term follow-up. ${ }^{14)}$ In our systematic review, the mean time from RTSA to diagnosis of acromial fracture was 9.4 months (ranges, 1 to 94 months). ${ }^{6-16,18-20)}$ Although Teusink et al. ${ }^{18)}$ reported a case of scapular spine fracture that occurred 94 months after RTSA, we found that most acromial and scapular spine fractures occurred within 24 months after RTSA. If a patient with RTSA complains of shoulder pain and limited ROM during the early postoperative period, physicians should suspect the presence of postoperative acromial fracture. Computed tomography can be a useful diagnostic tool to prevent additional fracture displacement and provide proper treatment. ${ }^{23)}$

Factors associated with postoperative acromial fractures include osteoporosis, prosthesis design, surgical approach, screw position and length in the glenoid, and technical factors such as excessive deltoid tension. ${ }^{11,20-23)}$ In our systematic review, we performed a statistical analysis of the association between postoperative acromial fractures and the implant design. The incidence was significantly different between the prosthesis designs: $8.4 \%$ for the MGMH, $4.0 \%$ for the LGMH and $2.8 \%$ for MGLH. According to Giles et al., ${ }^{24)}$ the lateralized center of rotation is smaller in MGLH than in LGMH, the abductor moment arm is larger in MGLH, and forces required for active abduction 
Cho et al. Acromial Fracture after Reverse Shoulder Arthroplasty: A Systematic Review

Clinics in Orthopedic Surgery • Vol. 11, No. 4, $2019 \bullet$ www.ecios.org

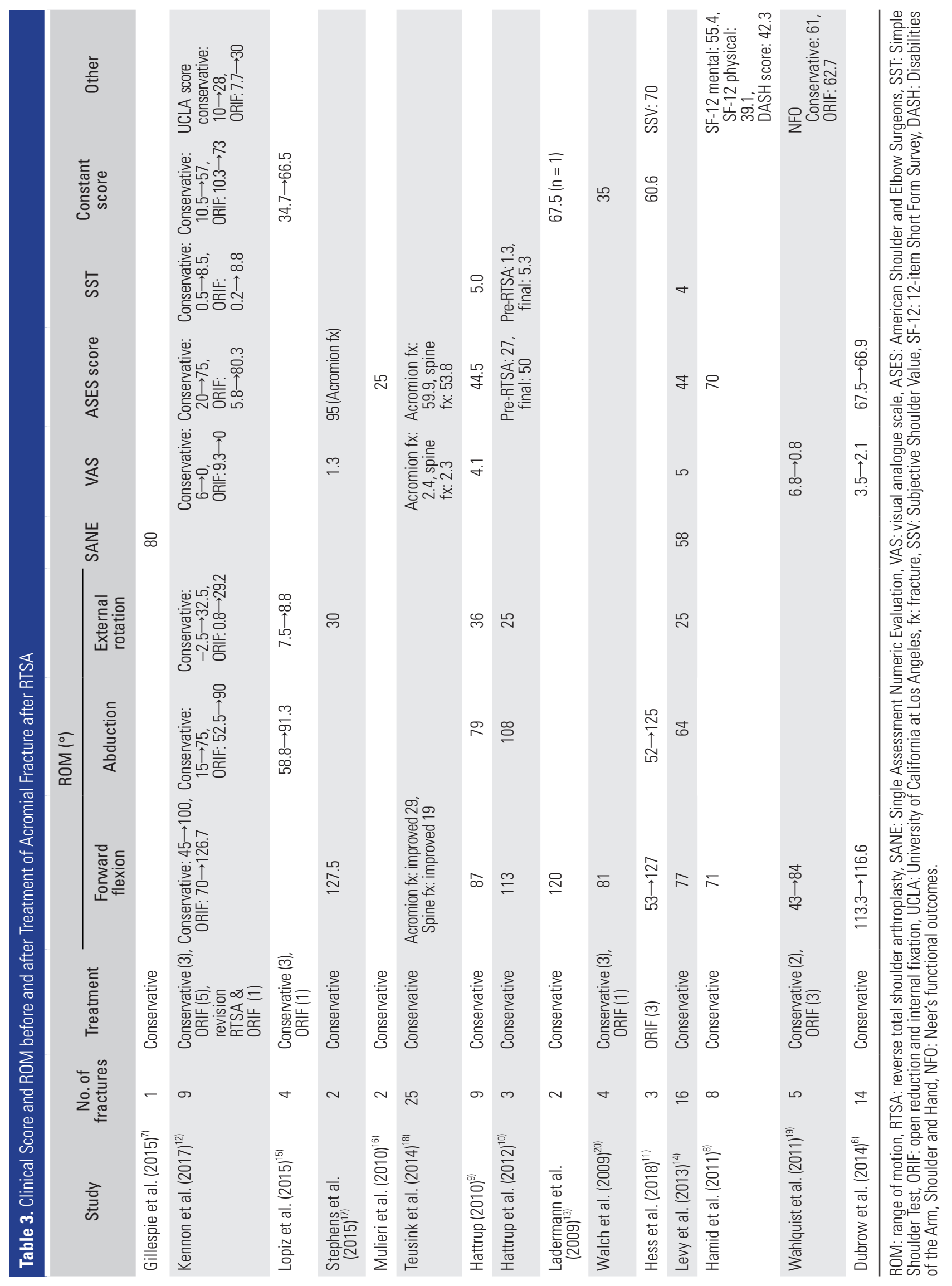


Cho et al. Acromial Fracture after Reverse Shoulder Arthroplasty: A Systematic Review

Clinics in Orthopedic Surgery • Vol. 11, No. 4, 2019• www.ecios.org

and joint load are significantly smaller in MGLH. Therefore, they suggested that the incidence of acromial fracture should be highest in MGMH and decrease in the order of LGMH and MGLH. ${ }^{24)}$ This was confirmed in our systematic review. However, we could not analyze the association between postoperative acromial fractures and other factors including osteoporosis, surgical technique, and screw position because of the lack of available data. Further largescale studies for identifying risk factors may help to reduce the incidence of acromial fracture after RTSA.

In our systematic review, the overall union rate was $50.0 \%$ (43.8\% for conservative treatment and $87.5 \%$ for operative treatment). However, we found no clear treatment guidelines for postoperative acromial fractures according to the displacement or location of fracture. The level of evidence was low in most articles included in this study, hence the lack of evidence-based treatment recommendations for the fracture. Furthermore, inconsistent results were reported after conservative treatment and operative treatment. $2,7,10,13,16,17,20)$

Acromial fractures after RTSA have varying effects on final outcomes. Boileau et al. ${ }^{2)}$ reported two acromial fractures that appeared as incidental findings 3 months after RTSA. Both patients were completely asymptomatic, and the fractures did not appear to have any detrimental effect on function. Dubrow et al. ${ }^{6}$ reported that most patients who had an acromial fracture appeared to be able to return to the previous level of postoperative function after conservative treatment. On the other hand, Walch et al. ${ }^{20)}$ reported that postoperative acromial fracture significantly worsened pain and function. Even after fracture union, postoperative acromial fractures can be associated with inferior outcomes. ${ }^{9,20)}$ Teusink et al. ${ }^{18)}$ reported that patients with a postoperative acromial fracture may be at increased risk for revision surgery and inferior outcomes compared with patients without a fracture. However, none of the studies that reported favorable outcomes after acromial fractures provided long-term follow-up results. Despite the presence of a report showing that acromial or scapular spine fractures lead to subsequent instability, it has not been elucidated whether revision rates are affected by postoperative fractures. ${ }^{14,18)}$

This study has several limitations. First, most of the included articles were case series with low quality of evidence. Our systematic review was based on level III and IV studies with low quality evaluation and short- to midterm follow-up. Second, data presentation and reported outcome measures were heterogeneous among the studies, which precluded statistical analysis of risk factors and outcomes. Therefore, it is difficult to draw any definitive conclusions regarding the difference in the outcomes between conservative and operative treatment. Further studies are required to identify fracture characteristics that determine the benefit from conservative or operative treatment.

In conclusion, acromial fractures after RTSA are a complication that is neither uncommon nor negligible. There is controversy on the outcomes after treatment of acromial fractures owing to the lack of studies with highlevel evidence. There are no clear treatment guidelines for acromial fractures after RTSA. Further well-designed prospective randomized controlled studies with a long-term follow-up should be performed to ascertain the diagnosis, treatment, and prognosis of acromial fractures after RTSA.

\section{CONFLICT OF INTEREST}

No potential conflict of interest relevant to this article was reported.

\section{ACKNOWLEDGEMENTS}

We thank Eun-Ji Jeon (Keimyung University Dongsan Medical Center) for her support with data collection.

\section{REFERENCES}

1. Grammont PM, Baulot E. Delta shoulder prosthesis for rotator cuff rupture. Orthopedics. 1993;16(1):65-8.

2. Boileau P, Watkinson DJ, Hatzidakis AM, Balg F. Grammont reverse prosthesis: design, rationale, and biomechanics. J Shoulder Elbow Surg. 2005;14(1 Suppl S):147S-161S.

3. Shafritz AB, Flieger S. Reverse total shoulder arthroplasty: early results of forty-one cases and a review of the literature. Hand Clin. 2012;28(4):469-79.

4. Bohsali KI, Wirth MA, Rockwood CA Jr. Complica- tions of total shoulder arthroplasty. J Bone Joint Surg Am. 2006;88(10):2279-92.

5. Saltzman BM, Chalmers PN, Gupta AK, Romeo AA, Nicholson GP. Complication rates comparing primary with revision reverse total shoulder arthroplasty. J Shoulder Elbow Surg. 2014;23(11):1647-54.

6. Dubrow S, Streit JJ, Muh S, Shishani Y, Gobezie R. Acromial stress fractures: correlation with acromioclavicular osteoarthritis and acromiohumeral distance. Orthopedics. 
Cho et al. Acromial Fracture after Reverse Shoulder Arthroplasty: A Systematic Review

Clinics in Orthopedic Surgery • Vol. 11, No. 4, 2019• www.ecios.org

2014;37(12):e1074-9.

7. Gillespie RJ, Garrigues GE, Chang ES, Namdari S, Williams GR Jr. Surgical exposure for reverse total shoulder arthroplasty: differences in approaches and outcomes. Orthop Clin North Am. 2015;46(1):49-56.

8. Hamid N, Connor PM, Fleischli JF, D'Alessandro DF. Acromial fracture after reverse shoulder arthroplasty. Am J Orthop (Belle Mead NJ). 2011;40(7):E125-9.

9. Hattrup SJ. The influence of postoperative acromial and scapular spine fractures on the results of reverse shoulder arthroplasty. Orthopedics. 2010;33(5):302.

10. Hattrup SJ, Sanchez-Sotelo J, Sperling JW, Cofield RH. Reverse shoulder replacement for patients with inflammatory arthritis. J Hand Surg Am. 2012;37(9):1888-94.

11. Hess F, Zettl R, Smolen D, Knoth C. Anatomical reconstruction to treat acromion fractures following reverse shoulder arthroplasty. Int Orthop. 2018;42(4):875-81.

12. Kennon JC, Lu C, McGee-Lawrence ME, Crosby LA. Scapula fracture incidence in reverse total shoulder arthroplasty using screws above or below metaglene central cage: clinical and biomechanical outcomes. J Shoulder Elbow Surg. 2017;26(6):1023-30.

13. Ladermann A, Williams MD, Melis B, Hoffmeyer P, Walch G. Objective evaluation of lengthening in reverse shoulder arthroplasty. J Shoulder Elbow Surg. 2009;18(4):588-95.

14. Levy JC, Anderson C, Samson A. Classification of postoperative acromial fractures following reverse shoulder arthroplasty. J Bone Joint Surg Am. 2013;95(15):e104.

15. Lopiz Y, Rodriguez-Gonzalez A, Garcia-Fernandez C, Marco F. Scapula insufficiency fractures after reverse total shoulder arthroplasty in rotator cuff arthropathy: what is their functional impact? Rev Esp Cir Ortop Traumatol. 2015; 59(5):318-25.
16. Mulieri P, Dunning P, Klein S, Pupello D, Frankle M. Reverse shoulder arthroplasty for the treatment of irreparable rotator cuff tear without glenohumeral arthritis. J Bone Joint Surg Am. 2010;92(15):2544-56.

17. Stephens SP, Paisley KC, Giveans MR, Wirth MA. The effect of proximal humeral bone loss on revision reverse total shoulder arthroplasty. J Shoulder Elbow Surg. 2015;24(10):1519-26.

18. Teusink MJ, Otto RJ, Cottrell BJ, Frankle MA. What is the effect of postoperative scapular fracture on outcomes of reverse shoulder arthroplasty? J Shoulder Elbow Surg. 2014;23(6):782-90.

19. Wahlquist TC, Hunt AF, Braman JP. Acromial base fractures after reverse total shoulder arthroplasty: report of five cases. J Shoulder Elbow Surg. 2011;20(7):1178-83.

20. Walch G, Mottier F, Wall B, Boileau P, Mole D, Favard L. Acromial insufficiency in reverse shoulder arthroplasties. J Shoulder Elbow Surg. 2009;18(3):495-502.

21. Crosby LA, Hamilton A, Twiss T. Scapula fractures after reverse total shoulder arthroplasty: classification and treatment. Clin Orthop Relat Res. 2011;469(9):2544-9.

22. Otto RJ, Virani NA, Levy JC, Nigro PT, Cuff DJ, Frankle MA. Scapular fractures after reverse shoulder arthroplasty: evaluation of risk factors and the reliability of a proposed classification. J Shoulder Elbow Surg. 2013;22(11):1514-21.

23. Mayne IP, Bell SN, Wright W, Coghlan JA. Acromial and scapular spine fractures after reverse total shoulder arthroplasty. Shoulder Elbow. 2016;8(2):90-100.

24. Giles JW, Langohr GD, Johnson JA, Athwal GS. Implant design variations in reverse total shoulder arthroplasty influence the required deltoid force and resultant joint load. Clin Orthop Relat Res. 2015;473(11):3615-26. 